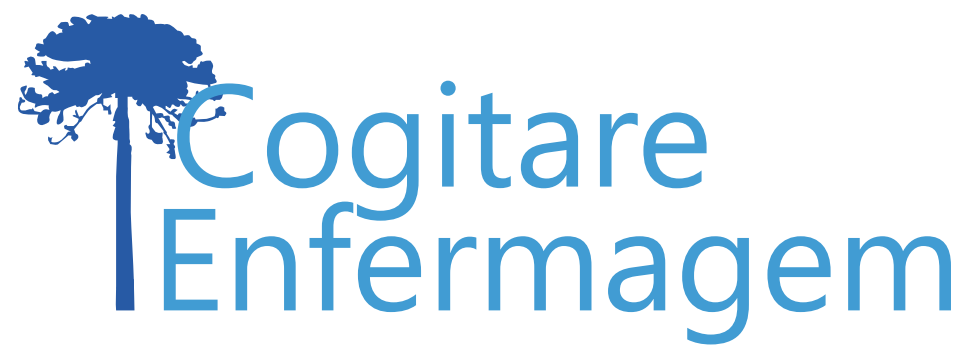

\title{
SAÚDE DO IDOSO EM TEMPOS DE PANDEMIA COVID-19
}

Karina Silveira de Almeida Hammerschmidt ${ }^{1}$, Rosimere Ferreira Santana ${ }^{2}$

\section{RESUMO}

Objetivo: trata-se de comunicação livre com intenção de abordar de forma reflexiva e crítica aspectos relacionados à saúde do idoso nos tempos de pandemia COVID-19.

Desenvolvimento: os idosos são destaque na pandemia COVID-19, especialmente aqueles com doenças crônicas e, com isso, ascendem-se preocupações com a diversidade do envelhecimento e com o idoso. Logo, as ações adotadas devem se fundamentar nos pilares da gerontologia, com manutenção da autonomia e independência; evitando ageísmo; com ajuste das ações de distanciamento social, impedindo a síndrome geriátrica de isolamento social. Não se verificou ênfase diferenciada para os idosos nos Protocolos de Manejo Clínico do Coronavírus, sendo essencial inclusão deste público nas diretrizes do Ministério da Saúde.

Considerações finais: urge a necessidade do Cuidado Gerontológico de Enfermagem robusto, qualificado e seguro, mediante fundamental capacitação profissional, sendo necessário ressignificar as ações de atenção ao idoso, respeitando a pluralidade, com foco no momento pandêmico e vislumbrando cenários futuros.

DESCRITORES: Enfermagem Geriátrica; Infecções por Coronavírus; Coronavírus; Geriatria; Idoso.

COMO REFERENCIAR ESTE ARTIGO:

Hammerschmidt KS de A, Santana RF. Saúde do idoso em tempos de pandemia Covid-19. Cogitare enferm. [Internet]. 2020 [acesso em "colocar data de acesso, dia, mês abreviado e ano"]; 25. Disponível em: http:// dx.doi.org/10.5380/ce.v25i0.72849.

Este obra está licenciado com uma Licença Creative Commons Atribuição 4.0 Internacional.

${ }^{1}$ Enfermeira. Doutora em Enfermagem. Docente de Enfermagem da Universidade Federal do Paraná. Curitiba, PR, Brasil. (9) ${ }^{2}$ Enfermeira. Doutora em Enfermagem. Docente de Enfermagem da Universidade Federal Fluminense. Rio de Janeiro, RJ, Brasil. 1 


\title{
HEALTH OF THE OLDER ADULTS IN TIMES OF THE COVID-19 PANDEMIC
}

\begin{abstract}
Objective: This is a free release with the intention to reflect and critically address aspects related to the health of older adults in the times of the COVID-19 pandemic.

Development: Older adults are in the spotlight in the COVID-19 pandemic, especially those with chronic diseases and, with this, concerns arise with the diversity of aging and with the older population. Thus, the actions adopted must be based on the pillars of gerontology, maintaining autonomy and independence; avoiding ageism; with adjustment of social distance actions, preventing the geriatric syndrome of social isolation. There was no specific emphasis for the older adults in the Coronavirus Clinical Management Protocols, being essential to include this population in the Brazilian Ministry of Health's guidelines.

Final considerations: There is an urgent need for robust, qualified, and safe Gerontological Nursing Care by means of essential professional training, and it is necessary to reframe the actions of care for the older adults, respecting their plurality, focusing on the pandemic moment, and envisioning future scenarios.
\end{abstract}

DESCRIPTORS: Geriatric Nursing; Coronavirus Infections; Coronavirus; Geriatrics; Older Adults.

\section{LA SALUD DE LAS PERSONAS MAYORES EN TIEMPOS DE LA PANDEMIA DEL COVID-19}

\begin{abstract}
RESUMEN:
Objetivo: el presente es un comunicado de libre difusión que pretende abordar de manera reflexiva y crítica diversos aspectos relacionados con la salud de las personas mayores en los tiempos de la pandemia del COVID-19.

Desarrollo: las personas mayores son el foco de atención en la pandemia del COVID-19, especialmente quienes padecen enfermedades crónicas $y$, con ello, surgen inquietudes con respecto a la diversidad del envejecimiento y las personas mayores. En consecuencia, las acciones que se adopten deben fundamentarse en los pilares de la gerontología, con la debida conservación de la autonomía y de la independencia; evitando la discriminación basada en la edad (o "edadismo"); con un ajuste en las acciones de distanciamiento social, impidiendo así la aparición del síndrome geriátrico del aislamiento social. No se verificó énfasis diferenciado alguno en relación con las personas mayores en los Protocolos de Manejo Clínico del Coronavirus, siendo esencial que se incluya a esta población en las directrices del Ministerio de Salud.

Consideraciones finales: urge la necesidad de implementar Cuidados Gerontológicos de Enfermería robustos, calificados y seguros, por medio de una capacitación profesional fundamental, con la necesidad de resignificar las acciones de atención para las personas mayores, respetando la pluralidad, con el movimiento pandémico como centro de atención, $y$ vislumbrando escenarios futuros.
\end{abstract}

DESCRIPTORES: Enfermería geriátrica; Infecciones por coronavirus; Coronavirus; Geriatría; Personas mayores. 
O novo coronavírus designado como Severe Acute Respiratory Syndrome Coronavirus-2 (Sars-Cov-2)(1), e como Corona Virus Disease-19 (COVID-19) para a doença ${ }^{(2,3)}$, promove infecção aguda, não há estado crônico de infecção e os seres humanos não são seus hospedeiros naturais; em 2 a 4 semanas, o vírus é eliminado pelo corpo humano; se o vírus não encontrar hospedeiro, a doença encerra-se; deste modo, o sucesso do combate depende da inflexão da pandemia ${ }^{(2-4)}$.

O primeiro caso do novo coronavírus foi notificado em Wuhan, na China, em 31 de dezembro de 2019 e foi declarada a Pandemia Mundial no dia 11 de março de 2020(5), responsável por mais de 1.350 .523 casos confirmados no total e 74.856 mortes no mundo até a data de 06 de abril de 2020. No Brasil, o primeiro caso positivo foi anunciado em 26 de fevereiro de 2020, sendo um homem morador de São Paulo, de 61 anos, que esteve na Itália(6). O primeiro óbito brasileiro confirmado ocorreu em 17 de março de 2020, com um homem de 62 anos, diagnosticado com diabetes e hipertensão, internado em rede especializada de saúde para a população idosa(7). Portanto, os idosos estão no centro da discussão da pandemia COVID-19 e carecem de atenção especializada de enfermagem e saúde para minimizar efeitos desastrosos no sistema de saúde e sociedade.

No mundo, no ano de 2020, há 1,1 bilhão de idosos, com projeção de 3,1 bilhões em 2100, o que converge com o cenário brasileiro, que apresenta 29,9 milhões em 2020 e previsão de 72,4 milhões em $2100^{(8)}$. Os dados do COVID-19 apontam maior taxa de mortalidade entre as pessoas com 80 anos ou mais, em que 14,8\% dos infectados morreram, comparado a $8,0 \%$ entre os idosos de 70 a 79 anos e 8,8\% entre aqueles de 60 a 69 anos (taxa 3,82 vezes maior que a média geral) ${ }^{(5,9,10)}$, reforçando as apreensões com a população idosa.

O risco de morrer de COVID-19 aumenta com a idade, já que a maioria das mortes ocorrem em idosos, especialmente aqueles com doenças crônicas ${ }^{(2,11)}$. A imunossenescência aumenta a vulnerabilidade às doenças infectocontagiosas e os prognósticos para aqueles com doenças crônicas são desfavoráveis ${ }^{(2,12)}$.

Durante essa pandemia, o mundo e o Brasil adotaram medidas de isolamento e distanciamento social, interrupção de aulas e trabalhos presenciais; gerando, além do sofrimento com o noticiário de mortes e hospitalizações, danos emocionais e financeiros ${ }^{(2)}$, que afetaram diferentemente cada grupo geracional. Portanto, para além das questões fisiopatológicas e epidemiológicas, há de se discutir o impacto da pandemia COVID-19 na saúde integral do idoso, família, profissionais de saúde e sociedade.

Esta comunicação livre tem intenção de abordar de forma reflexiva e crítica aspectos relacionados à saúde do idoso nos tempos de pandemia COVID-19.

\section{DESENVOLVIMENTO}

\section{Preocupações com os idosos}

Os idosos são destaque na pandemia COVID-19, em grande parte por apresentar alterações decorrentes da senescência ou senilidade ${ }^{(13,14)}$. Apesar do envelhecimento populacional, infelizmente há pouca visibilidade e valorização dessa parcela da população. Verifica-se continuamente visão preconceituosa, estigmatizada e estereotipada, instigando o ageísmo, que legitima a idade cronológica como diferenciador de classes, inclusive com envolvimento de crenças e atitudes que ridicularizam o idoso ${ }^{(15)}$. A pandemia COVID-19 aflorou o destaque aos idosos, principalmente devido ao potencial de risco dessa população, com direcionamento de ações e estratégias de distanciamento social especificamente para esse grupo. 
As ações de proteção à pessoa idosa na pandemia incluíram a estratificação etária, que apesar de positiva como organização do serviço, reforçou os preconceitos da sociedade, mediante a criação de diversos vídeos, imagens, frases, músicas, com exposição dos idosos e supervalorização de características eminentemente negativas. Como exemplo, pode-se destacar o emblemático caso brasileiro do "carro do 'cata véio'", que além do ageísmo, evidencia a dificuldade dos idosos cumprirem o distanciamento social. Estas situações também afetaram as relações familiares, com conflitos intergeracionais, principalmente devido às medidas adotadas pelos familiares para impor o distanciamento social.

O aprendizado reforçado nesta pandemia é que os idosos possuem características e peculiaridades próprias, além da diversidade/pluralidade/complexidade do envelhecimento humano. Nesse cenário, apesar dos conceitos fundamentais da epidemiologia, virologia, imunologia, e tantos outros necessários e recorrentes, não se pode abster dos fundamentos da teoria e prática gerontológica, que promovem o diferencial para a adoção de medidas eficazes na proteção do grupo de risco dos idosos.

A citar o exemplo do distanciamento social, determinado como medida para evitar a propagação da COVID-19(16), o qual poderia ter sido discutido em âmbito político-ministerial com apoio da gerontologia, visando minimizar seus efeitos nos hábitos rotineiros dos idosos $^{(17)}$; evitando o isolamento social considerado como síndrome geriátrica ${ }^{(18)}$; e os medos que envolvem o morrer ${ }^{(19)}$. Provavelmente quem atua com idosos, ou possui familiares nesta condição, presenciou situações com a população idosa reticente ao distanciamento social, o que reforça a necessidade de compreender as medidas para contenção da disseminação e tratamento da COVID-19 considerando as especificidades da área gerontológica.

Outra preocupação é a configuração dos arranjos familiares. Há idosos que residem sozinhos ou com o cônjuge e outros com descendentes, envolvendo ambiente familiar cogeracional ativo ou passivo para apoio social. Os agentes que compõem o conjunto familiar podem incluir filhos, netos, bisnetos, cônjuges e outros membros; o apoio recebido pode ser instrumental (direcionado às atividades básicas, instrumentais e avançadas de vida diária), emocional (envolvendo atenuar o estresse) e/ou financeiro ${ }^{(20)}$.

$\mathrm{Na}$ coabitação, os idosos podem assumir papel de cuidador de outros, incluindo crianças, adolescentes, adultos ou idosos dependentes. Isso requer habilidades para atender às particularidades de diferentes gerações, assim como sobrecarga. Há maior nível de sobrecarga em cuidadores idosos que moram com crianças, principalmente devido à obrigatoriedade do cuidado diário(21). Entre os cuidados orientados durante a pandemia COVID-19, está o distanciamento principalmente das crianças, devido ao potencial de transmissão destas ${ }^{(4)}$.

Outra inquietação envolve os idosos institucionalizados. Estudos preliminares apontam que, nestas realidades, a infecção pelo SARS-CoV-2 é alta, com sugestão de taxa de mortalidade para maiores de 80 anos superior a $15 \%{ }^{(22)}$. Este contexto é considerado de alto risco para infecção, pois envolve predominantemente idosos, diversos com comorbidades crônicas e dificuldades para atividades da vida diária; contato frequente de cuidadores, profissionais e visitantes; e convivência em aglomerados ${ }^{(12)}$.

Também paira a preocupação com os idosos trabalhadores, os quais em diversas famílias representam a única fonte de renda. No Brasil, muitos idosos têm esta responsabilidade financeira(23), apesar do senso comum estimá-los como aposentados e pensionistas.

Outra hesitação é o estímulo do idoso para utilizar ferramentas tecnológicas. Essas podem possibilitar aproximação social, porém, historicamente, a população idosa brasileira apresenta baixa escolaridade e dificuldade de acesso aos recursos tecnológicos. Infelizmente, este fato interfere na aquisição de conhecimentos sobre a pandemia, assim como limita as possibilidades de comunicação, principalmente durante o distanciamento, dificultando a orientação dos comportamentos individuais e $\operatorname{coletivos}^{(3,5)}$. 


\section{Comportamento preventivo individual e coletivo}

Entre as diretrizes recomendadas para segurança dos idosos durante a pandemia, está o distanciamento e isolamento social(4,5). O distanciamento social denota a necessidade de (re)configuração dos comportamentos, com prioridade para ações de higiene constantes, como lavagem das mãos, uso de álcool em gel, distanciamento de outras pessoas, etiqueta respiratória, cuidados ambientais e emocionais ${ }^{(24)}$. Neste ínterim, a família e a sociedade podem se tornar um sistema de apoio ao idoso ${ }^{(20)}$; recomenda-se relacionamento permeado por respeito, verdade, informação, pactuação conjunta de atividades diárias e apoio.

É emergente definir e defender que o distanciamento social não caracteriza abandono, portanto, cada família em conjunto com o idoso precisa refletir e discutir as estratégias importantes para seu contexto. Neste momento de pandemia COVID-19, o afastamento físico reflete ato de amor, carinho e consideração, além de ser estratégia de proteção.

Outro comportamento preventivo é a conscientização sobre a vacinação, considerada conquista da humanidade para controle e erradicação de doenças infectocontagiosas ${ }^{(25)}$. Apesar dos investimentos, até o momento não há vacina para a COVID-19, porém no Brasil, devido ao período de ocorrência da pandemia, outras vacinas são relevantes, como a da Influenza. Neste ano (2020), a procura por essa vacina foi maciça, com esgotamento das doses em horas em algumas cidades e dias no território nacional ${ }^{(7)}$.

A vacinação, apesar de ser procedimento com o objetivo de provocar reação benéfica no organismo, pode resultar em eventos adversos pós-vacinação, os quais podem estar relacionados à contaminação, adulteração, ou a outros problemas decorrentes do processo de produção e ou aplicação ${ }^{(25)}$. No Brasil no ano de 2020 , as dificuldades durante a campanha Influenza envolveram a procura extensa e inesperada dos idosos, além dos ambientes compartilhados. Desse modo, o Ministério da Saúde rapidamente reorganizou a vacinação por subestratificação etária, possibilitando reforço na credibilidade da vacina e responsabilidade solidária da imunização em tempos de pandemia COVID-19.

Essa estratégia de vacinação antecipada e agrupada dos idosos, apesar de necessária, demonstrou novamente a necessidade de reconhecimento do padrão de comportamento do idosos. A contradição entre o pedido para o distanciamento e a chamada para a campanha de vacinação fez estímulo gatilho para os idosos saírem de suas casas, contradizendo as recomendaç̃es dos profissionais de saúde para não permanecerem nas ruas e nos serviços de saúde. Além disso, a propagação de fake news estimulou a compreensão por parte dos idosos de que estariam tomando a vacina contra a COVID-19.

Os dados estatísticos e comportamentos individuais e coletivos durante essa pandemia reforçam a essencialidade de valorizar os seres humanos ${ }^{(26)}$. Para tanto, o sistema de saúde precisa estar preparado para o cuidado ao idoso, em tempo de pandemia e fora deste, sendo imprescindível desenvolver estratégias alinhadas ao cuidado gerontológico de enfermagem seguro e qualificado.

\section{Cuidado gerontológico de enfermagem}

Para deter a pandemia, é essencial controlar a fonte de infecção, interromper a rota de transmissão e proteger as pessoas suscetíveis ${ }^{(2)}$. Nessa compreensão, durante a crise do novo coronavírus, vários profissionais de saúde se destacaram pelo seu trabalho, entre esses a Enfermagem, que está atuando na linha de frente. Essa fase da luta contra o novo coronavírus não envolve apenas coragem, mas racionalidade, paciência e ciência (2), deste modo, o cuidado gerontológico de enfermagem é primordial enquanto fortaleza dá ciência ${ }^{(27,28)}$.

Durante o período da pandemia, verificou-se entre os profissionais que atuam no cuidado à saúde ampla busca por conhecimentos, inclusive com documentos e cursos ofertados gratuitamente pelo Ministério da Saúde, Universidades Federais e Privadas, Sociedades Científicas e outras Instituições ${ }^{(24,29,30)}$. Porém, principalmente os materiais e 
documentos oficiais do Ministério da Saúde apresentaram pouca ênfase ao público idoso, predominando questões envolvendo crianças, adultos e gestantes. Diante da complexidade do processo de envelhecimento humano, com peculiaridades próprias, aliada à alta incidência das doenças crônicas e suas repercussões no corpo humano, evidencia-se a necessidade de atenção específica aos idosos, incluindo ações de prevenção, tratamento e reabilitação(31).

Para isso, a formação qualificada de enfermagem é destaque: investir nesses profissionais contribuirá nã̃o apenas para saúde, mas também para a educação, trabalho e crescimento econômico ${ }^{(30)}$. Além do enfoque global da enfermagem, com valorização de sua atuação na pandemia, o ano de 2020 foi designado pela $72^{\circ}$ Assembleia Mundial da Saúde como Ano Internacional da Enfermagem; convergindo estratégia da Agenda Mundial da Enfermagem para 2030, que referenda investir no ensino de enfermagem (corpo docente, infraestrutura e estudantes), para atender às necessidades globais, demandas domésticas e responder às mudanças tecnológicas e modelos avançados de saúde e assistência social integrados ${ }^{(30)}$.

Certamente, a formação especializada em enfermagem gerontológica terá transformações profundas pós-pandemia COVID-19, pois vivenciou-se na prática a essencialidade do cuidado gerontológico de enfermagem, fortalecido, robusto e preparado para as necessidades emergentes e reemergentes.

A demanda do cuidado gerontológico ficou evidente junto aos idosos institucionalizados, devido à vulnerabilidade destes durante a pandemia. Desta forma, para atender a demanda nas Instituições de Longa Permanência para Idosos (ILPI), a Associação Brasileira de Enfermagem, por meio do Departamento Científico de Enfermagem Gerontológica, elaborou documento ${ }^{(32)}$ com orientações específicas para essas instituições. A Sociedade Brasileira de Geriatria e Gerontologia também desenvolveu posicionamento sobre COVID-19(33); e a Agência Nacional de Vigilância Sanitária (ANVISA) emitiu nota técnica com orientações para serviços de saúde ${ }^{(34)}$ e outra nota específica para ILP|(1).

\section{Cenários futuros: ressignificar relações e ações}

A vivência da pandemia COVID-19 ressignificou condutas, conhecimentos e aproximou a comunidade do meio científico, pois, para o controle, são necessárias mudanças comportamentais individuais e coletivas, porém, não unicamente durante o período de pandemia. Os aprendizados sobre higiene das mãos, etiqueta respiratória, limpeza de ambientes e materiais ${ }^{(35)}$ devem ser incorporados cotidianamente, como atitudes de cooperação coletiva, pois são fortes medidas para prevenção de novas doenças. É essencial que as experiências propiciadas por este turbulento momento de crise fortaleçam e preparem a sociedade para outras situações de tensão.

Outro fator de destaque no aprendizado deste período e projeção para cenários futuros é o cuidado e atenção com os idosos, mediante estratégias de apoio e alerta para sinais e sintomas. Mesmo aqueles que residem sozinhos precisam contar com pessoas de referência para reportar suas necessidades, sentimentos ou relatos de saúde e doença. Além de ser oportuno ressignificar os vínculos com os idosos, também são importantes atitudes de respeito e consideração com este público.

Os momentos de distanciamento social são medidas de precaução à saúde, com intenção de preservação e proteção; porém, a autonomia e a independência do idoso são alicerces para o envelhecimento saudável. Desse modo, é necessário liberdade para exercê-las, respeitando o que é possível quando recomendado. A sociedade precisa ter atenção para evitar atitudes de preconceito, que infantilizam e ridicularizam o idoso. Não se pode retroceder em relação a este aspecto; a dignidade ao idoso deve prevalecer.

Também merece ênfase a incessante busca dos profissionais e população pelo conhecimento, durante o período da pandemia COVID-19. Diversos ambientes virtuais de aprendizagem foram desenvolvidos e/ou disponibilizados, com variados conteúdos e 
linguagem. A valorização oportuna, com destaque ao ensino geriátrico e gerontológico na formação profissional, deve ser item obrigatório na matriz curricular de enfermagem. O conhecimento é essencial para a evolução e, no âmbito profissional, é necessário para qualificação e segurança do cuidado ao idoso. Espera-se que esse movimento de aprendizado seja constante e perene, fortalecendo a enfermagem gerontológica enquanto ciência.

\section{CONSIDERAÇÕES FINAIS}

A pandemia COVID-19 destacou a saúde dos idosos, incitando necessidade de proteção, respeito, zelo, dignidade e rede de apoio, porém também aflorou ageísmo, julgamentos e ridicularização. A ação protetiva de distância deve manter a autonomia e a independência do idoso, e os profissionais necessitam de conhecimento específico sobre esta população.

O momento pandêmico reforçou a necessidade do Cuidado Gerontológico de Enfermagem robusto, qualificado e seguro, mediante fundamental capacitação profissional, sendo necessário ressignificar as ações de atenção ao idoso, respeitando a pluralidade e vislumbrando tanto o presente quanto os cenários futuros.

\section{REFERÊNCIAS}

1. Agência Nacional de Vigilância Sanitária (ANVISA). Nota técnica n. 05/2020 GVIMS/GGTES. Orientações para a Prevenção e Controle de Infecções pelo novo coronavírus (SARS-Cov-2) em Instituições de Longa Permanência para Idosos (ILPI). Brasília, 24 de março de 2020. [Internet]. 2020. [acesso em 02 abr. 2020]. Disponível em: http://portal.anvisa.gov.br/documents/33852/271858/ NOTA+T\%C3\%89CNICA+N\%C2\%BA+05-2020+GVIMS-GGTES-ANVISA+-+ORIENTA\%C3\%87\%C3\% 95ES+PARA+A+PREVEN\%C3\%87\%C3\%830+E+O+CONTROLE+DE+INFEC\%C3\%87\%C3\%95ES+PE LO+NOVO+CORONAV\%C3\%8DRUS+EM+INSTITUI\%C3\%87\%C3\%95ES+DE+LONGA+PERMAN\%C3\%8 ANCIA+PARA+IDOSOS\%28ILPI\%29/8dcf5820-fe26-49dd-adf9-1cee4e6d3096.

2. Zhang, W. Manual de Prevenção e Controle da Covid-19 segundo o Doutor Wenhong Zhang. São Paulo: PoloBooks; 2020.

3. Ministério da Saúde (BR). O que é o Coronavírus? (COVID-19). [Internet]. 2020. [acesso em 22 mar 2020]. Disponível em: https://coronavirus.saude.gov.br/.

4. Ministério da Saúde (BR). Secretaria de Atenção Especializada à Saúde. Departamento de Atenção Hospitalar, Domiciliar e de Urgência. Protocolo de Tratamento do Novo Coronavírus (2019-nCoV). [Internet]. Brasília: Ministério da Saúde; 2020 [acesso em 02 abr 2020]; Disponível: https://portalarquivos2. saude.gov.br/images/pdf/2020/fevereiro/05/Protocolo-de-manejo-clinico-para-o-novo-coronavirus-2019ncov.pdf.

5. World Health Organization (WHO). Novel Coronavirus (2019-nCoV) technical guidance, 2020. [Internet]. Geneva: WHO; 2020 [acesso em 04 abr 2020]. Disponível em: https://www.who.int/emergencies/ diseases/novel-coronavirus-2019.

6. Ministério da Saúde (BR). Secretaria de Vigilância em Saúde. Boletim Epidemiológico. Situação epidemiológica da febre amarela no monitoramento 2019/2020. [Internet]. Brasília: Ministério da Saúde; 2020. [acesso em 04 abr 2020]. Disponível em: https://www.saude.gov.br/images/pdf/2020/janeiro/15/ Boletim-epidemiologico-SVS-01.pdf.

7. Ministério da Saúde (BR). Secretaria de Vigilância em Saúde. Boletim Epidemiológico. Infecção Humana pelo Novo Coronavírus (2019-nCoV). [Internet]. Brasília: Ministério da Saúde; 2020. [acesso em 31 mar 2020]. Disponível em: https://www.saude.gov.br/images/pdf/2020/fevereiro/04/Boletim-epidemiologicoSVS-04fev20.pdf. 
8. World Health Organization (WHO). Elder abuse. [Internet]. Geneva: WHO; 2018. [acesso em 05 abr 2020]; Disponível em: https://www.who.int/en/news-room/fact-sheets/detail/elder-abuse.

9. Centers For Disease Control And Prevention. [Internet]. 2020. [acesso em 08 abr. 2020]; Disponível em: https://www.cdc.gov/coronavirus/2019-nCoV/hcp/infection-control.html.

10. Chen N, Zhou M, Dong X, Qu J, Gong F, Han Y, et al. Epidemiological and clinical characteristics of 99 cases of 2019 novel coronavirus pneumonia in Wuhan, China: a descriptive study. The Lancet. [Internet]. 2020. [acesso em 31 mar. 2020]; 395(10223). Disponível em: https://www.ncbi.nlm.nih.gov/ pubmed/32007143.

11. Lloyd-Sherlock P, Ebrahim S, Geffen L, Mckee M. Bearing the brunt of covid-19: older people in low and middle income countries. BMJ. [Internet]. 2020 [acesso em 31 mar 2020]; 368. Disponível em: https:// doi.org/10.1136/bmj.m1052.

12. Nunes VM de A, Machado FC de A, Morais MM de, Costa L de A, Nascimento ICS do, Nobre TTX, et al. COVID-19 e o cuidado de idosos: recomendações para instituições de longa permanência. Natal: EDUFRN; 2020. [Internet]. 2020. [acesso em 02 abr 2020]. Disponível em: https://repositorio.ufrn.br/jspui/ handle/123456789/28754.

13. Ministério da Saúde (BR). Secretaria de Vigilância em Saúde. Boletim Epidemiológico. Doença pelo Coronavírus 2019. [Internet]. Brasília: Ministério da Saúde; 2020. [acesso em 04 abr 2020]. Disponível em: https://portalarquivos.saude.gov.br/images/pdf/2020/April/03/BE6-Boletim-Especial-do-COE.pdf.

14. Valença TDC, Santos W da S, Lima PV, Santana E dos S, Reis LA dos. Deficiência física na velhice: um estudo estrutural das representações sociais. Esc. Anna Nery. [Internet]. 2017. [acesso em 05 abr 2020]; 21(1). Disponível em: http://www.scielo.br/scielo.php?script=sci arttext\&pid=S1414$81452017000100208 \&$ lng $=p t \& n r m=i s o$.

15. Goldani AM. "Ageism" in Brazil: what is it? who does it? what to do with it?. Rev. bras. estud. popul. [Internet]. 2010. [acesso em 05 abr 2020]; 27(2). Disponível em: http://www.scielo.br/scielo. php? script=sci arttext\&pid=S0102-30982010000200009\&lng=en\&nrm=iso.

16. Ministério da Saúde (BR). Secretaria de Vigilância em Saúde. Boletim Epidemiológico. Especial: doença pelo Coronavírus 2019. [Internet]. 2020. [acesso em 04 abr 2020]. Disponível em: https://www. saude.gov.br/images/pdf/2020/April/06/2020-04-06---BE7---Boletim-Especial-do-COE---Atualizacao-daAvaliacao-de-Risco.pdf.

17. Santos GLA, Santana RF. Capacidade funcional de idosos: implicações para atividades instrumentais de vida diária. Rev RENE. [Internet]. 2017 [acesso em 10 abr 2020]; 18(5). Disponível em: http:// periodicos.ufc.br/rene/article/view/30820.

18. Hughes LD, Mcmurdo MET, Guthrie B. Guidelines for people not for diseases: the challenges of applying UK clinical guidelines to older people with multimorbidity. Age Ageing. [Internet]. 2013 [acesso em 09 abr 2020]; 42(1). Disponível em: https://www.ncbi.nlm.nih.gov/pubmed/22910303.

19. Von Hohendorf J, Melo WV de. Compreensão da morte e desenvolvimento humano: contribuições à psicologia hospitalar. Estud. pesqui. psicol. [Internet]. 2009 [acesso em 09 abr 2020]; 9(2). Disponível em: http://pepsic.bvsalud.org/scielo.php?script=sci arttext\&pid=S1808-42812009000200014\&lng=pt\&nrm=i so.

20. Oliveira MCGM de, Salmazo-Silva H, Gomes L, Moraes CF, Alves VP. Elderly individuals in multigenerational households: family composition, satisfaction with life and social involvement. Estud. psicol. (Campinas). [Internet]. 2020. [acesso em 05 abr 2020]; 37(e180081). Disponível em: http://www. scielo.br/scielo.php?script=sci arttext\&pid=S0103-166X2020000100800\&lng=pt\&nrm=iso.

21. Oliveira NA de, Souza EM, Luchesi BM, Alexandre T da S, Inouye K, Pavarini SCI. Elderly caregivers of other elderly living with and without children: burden, optimism and coping strategies. Cienc. saude colet. [Internet]. 2020. [acesso em 05 abr 2020]; 25(2). Disponível em: http://www.scielo.br/scielo. php?script=sci arttext\&pid=S1413-81232020000200473\&lng=pt\&nrm=iso. 
22. Ouslander JG. Coronavirus Disease19 in Geriatrics and Long-Term Care: an update. Editorial. J Am Geriatr Soc. [Internet]. 2020 [acesso em 03 abr 2020]. Disponível em: https://doi.org/10.1111/jgs.16464.

23. Faustino CG, Levy RB, Canella DS, Oliveira C de, Novaes HMD. Income and out-of-pocket health expenditure in living arrangements of families with older adults in Brazil. Cad. Saude Publica. [Internet]. 2020 [acesso em 05 abr 2020]; 36(3). Disponível em: https://doi.org/10.1590/0102-311x00040619.

24. Ministério da Saúde (BR). Portaria n. 639, de 31 de março de 2020. Dispõe sobre a Ação Estratégica "O Brasil Conta Comigo - Profissionais da Saúde", voltada à capacitação e ao cadastramento de profissionais da área de saúde, para o enfrentamento à pandemia do coronavírus (COVID-19). [Internet]. Diário Oficial da República Federativa do Brasil. 02 abr 2020. [acesso em 02 abr 2020]. Disponível em: http://www.in.gov.br/en/web/dou/-/portaria-n-639-de-31-de-marco-de-2020-250847738.

25. Lessa S de C, Schramm FR. Proteção individual versus proteção coletiva: análise bioética do programa nacional de vacinação infantil em massa. Cienc. saude colet. [Internet]. 2015 [acesso em 05 abr 2020]; 20(1). Disponível em: https://doi.org/10.1590/1413-81232014201.14882013.

26. Cesari M, Proietti M. Geriatric medicine in italy in the time of COVID-19. J Nutr Health Aging. [Internet]. 2020. [acesso em 02 abr 2020]: 24. Disponível em: https://www.ncbi.nlm.nih.gov/pmc/articles/ PMC7118363/.

27. Barros ALBL de, Nobrega MML da, Santos R da S, Cezar-Vaz MR, Pagliuca LMD. Pesquisa em enfermagem e a modificação da árvore do conhecimento no CNPq: contribuição à ciência. Rev. bras. enferm. [Internet]. 2020. [acesso em 08 abr 2020]; 73(1). Disponível em: https://doi.org/10.1590/00347167-2017-0911.

28. Ministério da Saúde (BR). Secretaria de Atenção Primária à Saúde (SAPS). Procedimento Operacional Padronizado: equipamento de proteção individual e segurança no trabalho para profissionais de saúde da APS no atendimento às pessoas com suspeita ou infecção pelo novo Coronavírus (COVID-19). [Internet]. Brasília: Ministério da Saúde: 2020. [acesso em 01 abr 2020]. Disponível em: https://www.unasus.gov.br/ especial/covid19/pdf/67.

29. Ministério da Saúde (BR). Secretaria de Ciência, Tecnologia, Inovação e Insumos Estratégicos em Saúde. Diretrizes para diagnóstico e tratamento da COVID-19. [Internet]. Brasília: Ministério da Saúde; 2020. [acesso em 07 abr 2020]. Disponível em: https://portalarquivos.saude.gov.br/images/pdf/2020/ April/07/ddt-covid-19.pdf.

30. World Health Organization (WHO). State of the world's nursing 2020: investing in education, jobs and leadership. [Internet]. Geneva: WHO; 2020. [acesso em 08 abr 2020]. Disponível em: https://apps.who.int/ iris/bitstream/handle/10665/331677/9789240003279-eng.pdf?sequence=1\&isAllowed=y.

31. Tristão FR, Girondi JBR, Hammerschmidt KS de A, Zamprogna KM, Soares CF, Evaristo SM, et al. Práticas de cuidados do enfermeiro na atenção primária à saúde: gestão do cuidado da pele do idoso. Cogitare enferm. [Internet]. 2020. [acesso em 10 abr 2020]; (25). Disponível em: https://revistas.ufpr.br/ cogitare/article/view/65223.

32. Associação Brasileira De Enfermagem (ABEn Nacional). Departamento Científico de Enfermagem Gerontológica. Comunicação aos trabalhadores de enfermagem das instituições de longa permanência de idosos (ILPI) para o enfrentamento da disseminação da COVID-19. [Internet]. 2020. [acesso em 05 abr 2020]. Disponível em: http://www.abennacional.org.br/site/wp-content/uploads/2020/03/DCEG-ABEn Informe COVID-19-ILPI.pdf.

33. Uehara CA, Berkenbrock I, Herédia VBM, Bandeira R, Kairalla M, Gomes DA, et al. Posicionamento sobre COVID-19. Sociedade Brasileira de Geriatria e Gerontologia - SBBG. [Internet]. 2020. [acesso em 08 abr 2020]. Disponível em: https://sbgg.org.br/posicionamento-sobre-covid-19-sociedade-brasileira-degeriatria-e-gerontologia-sbgg-atualizacao-15-03-2020/.

34. Agência Nacional de Vigilância Sanitária (ANVISA). Nota técnica n. 04/2020 GVIMS/GGTES. Apresenta orientações para serviços de saúde: medidas de prevenção e controle que devem ser adotadas durante a assistência aos casos suspeitos ou confirmados de infecção pelo novo Coronavírus 
(COVID-19). [Internet]. 2020. [acesso: 02 abr. 2020]. Disponível em: http://portal.anvisa.gov.br/ documents/33852/271858/Nota+T\%C3\%A9cnica+n+04-2020+GVIMS-GGTES-ANVISA/ab598660-3de44f14-8e6f-b9341c196b28.

35. Organização Panamericana de Saúde (OPAS). Prevenção e controle de infecção durante os cuidados de saúde quando houver suspeita de infecção pelo novo Coronavírus (nCoV). Diretrizes provisórias 25 de janeiro 2020. [Internet]. 2020. [acesso em 10 abr 2020]. Disponível em: https://www.paho.org/bra/ index.php?option=com docman\&view=document\&layout=default\&alias=1918-prevencao-e-controlede-infeccao-durante-os-cuidados-de-saude-quando-houver-suspeita-de-infeccao-pelo-novo-coronavirusncov\&category slug=pasta-temporaria-periodo-de-transicao-no-iris-ate-22-2\&Itemid=965.

Recebido: $13 / 04 / 2020$

Finalizado: 23/04/2020

Editora associada: Luciana Puchalski Kalinke

Autor Correspondente:

Karina Silveira de Almeida Hammerschmidt

Universidade Federal do Paraná

Av. Prefeito Lothário Meissner, 632 - 80210-170 - Curitiba, PR, Brasil

E-mail: ksalmeidah@yahoo.com.br

Contribuição dos autores:

Contribuições substanciais para a concepção ou desenho do estudo; ou a aquisição, análise ou interpretação de dados do estudo - KSAH

Aprovação da versão final do estudo a ser publicado - RFS 